\title{
Comunicação
}

[Communication]

\section{Leveduras isoladas do pelame de cães sadios que vivem em regime domiciliar}

\author{
[Yeasts isolated from the haircoat of healthy dogs that live indoor] \\ H.D.L. Bentubo ${ }^{1}$, W. Gambale ${ }^{1}$, O. Fischman ${ }^{2}$ \\ ${ }^{1}$ Departamento de Microbiologia - ICB-USP - São Paulo, SP \\ ${ }^{2}$ Departamento de Microbiologia, Imunologia e Parasitologia - UNIFESP - São Paulo, SP
}

O contato prévio com o microrganismo constitui fator determinante para o estabelecimento de doenças infecciosas, condição limitante à vida dos animais de estimação. Fatores estressantes, como restrição ao exercício, brigas com rivais e longos períodos de isolamento, podem colaborar de forma relevante para a deterioração dos mecanismos naturais de resistência, aumentando ainda mais a susceptibilidade desses animais. A acurácia no diagnóstico e os novos protocolos terapêuticos têm proporcionado melhor prognóstico para portadores de doenças crônicodegenerativas ou alérgicas, que fazem uso regular de antimicrobianos e anti-inflamatórios potentes, às vezes por períodos prolongados, representando risco iminente de infecção oportunista para pacientes nessas condições. Além de destruir a população indígena de microrganismos, o uso crônico e indiscriminado desses medicamentos seleciona resistentes. Apenas o uso criterioso e o combate à comercialização indiscriminada e à medicação sem prescrição podem atenuar seus efeitos indesejados (Bentubo et al., 2007; Cleff et al., 2007).

De maneira geral, os fungos podem colonizar os tecidos de seres humanos e animais. A colonização e as enfermidades produzidas por fungos dermatófitos são bem conhecidas. No entanto, o mesmo não ocorre com as leveduras que podem, eventualmente, colonizar o corpo dos animais. Segundo a literatura, os principais gêneros de fungos associados com a colonização do pelame e cavidades naturais de cães e gatos são: Aspergillus spp., Cladosporium spp.,
Candida spp., Cryptococcus spp., Fusarium spp., Geotrichum spp., Malassezia spp., Microsporum spp., Penicillium spp., Rhodotorula spp., Scopulariopsis spp., Trichophyton spp., e Trichosporon spp. (Cabañes et al., 1996; Cleff et al., 2007). Os estudos de microbiota fúngica normal com caráter preditivo de infecções oportunistas são escassos. Em muitos casos, as espécies de leveduras encontradas na microbiota nem sempre são identificadas de forma adequada para que se obtenha interpretação segura a despeito do isolamento. Dessa forma, a interpretação clínica dos achados laboratoriais fica comprometida e pode induzir o clínico menos experiente a erro quando este se depara com quadros dermatológicos inespecíficos, principalmente os de etiologia infecciosa (Souza e Scarcelli, 2000). A relação existente entre a colonização e os fatores predisponentes de infecções oportunistas ainda é pouco discutida em medicina veterinária. Apesar dos avanços científicos e tecnológicos da área, os fatores que favorecem a colonização, a proliferação e a transmissão desses agentes ainda são pouco compreendidos, necessitando, portanto, de novos estudos que esclareçam melhor esses aspectos importantes (Cleff et al., 2007).

O objetivo da presente investigação foi isolar e identificar as espécies de leveduras, potencialmente patogênicas, que compõem a microbiota residente/transitória do tegumento de cães sadios domiciliados, discutindo aspectos epidemiológicos relacionados com as infecções fúngicas oportunistas.

Recebido em 10 de outubro de 2009

Aceito em 2 de agosto de 2010

E-mail: hbentubo@yahoo.com.br 
Foram estudados 21 cães adultos, sadios, de diversas raças, de ambos os sexos, que viviam em estreito contato com seus proprietários e frequentavam com regularidade serviço de estética canina, na cidade de São Paulo. As coletas foram realizadas mediante consentimento do responsável, e as amostras foram obtidas de acordo com a técnica descrita por Mariat e Adam-Campos (1967), que preconiza a fricção de quadrados de carpete esterilizados $(5 \times 5 \mathrm{~cm})$ no pelame dos animais. Após a coleta, as amostras foram acondicionadas em seu invólucro original e encaminhadas ao laboratório em até 12 horas para processamento. Cada carpete foi pressionado sobre placas de Petri contendo meio de ágar Sabouraud dextrose (Difco - Baltimore, EUA) acrescido de cloranfenicol (100mg/L), incubado a $35^{\circ} \mathrm{C}$ e observado diariamente quanto ao crescimento de microrganismos leveduriformes durante sete dias. O procedimento de coleta das amostras atendeu às normas estabelecidas pelo Comitê de Ética em Pesquisa Envolvendo Animais, do Instituto de Ciências Biomédicas da Universidade de São Paulo, São Paulo. Os isolados leveduriformes foram identificados pelas suas características morfológicas e bioquímicas (De Hoog et al., 2000). Meio comercial de CHROMagar Candida $^{\circledR}$ (Difco) foi empregado como meio adicional de identificação prévia dos isolados leveduriformes.

A população estudada era composta de 10 raças: Buldogue inglês (2), Coccker spaniel inglês (1), Lhasa apso (1), Mastiff inglês (1), Pinscher miniatura (1), Poodle (3), Retriever labrador (2), Rottweiler (1), Schnawzer miniatura (1), SRD (sem raça definida) (5) e Yokshire terrier (3). Destes, 12 eram fêmeas e nove eram machos, com idades entre 12 e 72 meses. Candida albicans foi isolada de $95,2 \%$ dos animais (20/21), enquanto $R$. mucilaginosa foi identificada em 4,8\% (1/21). Não foram obtidas culturas mistas. Todos os isolados de $C$. albicans apresentaram-se verdes quando semeados em meio de CHROMagar Candida ${ }^{\circledR}$.

A microbiota superficial dos mamíferos é composta por ampla variedade de agentes fúngicos. Os fungos são microrganismos amplamente encontrados na natureza, por isso a composição dessa microbiota pode ser bastante influenciada pelo meio ambiente. Candida sp. e Rhodotorula sp. foram isoladas da população estudada. Os resultados encontrados são semelhantes aos da literatura que cita o isolamento dos gêneros Candida, Cryptococcus, Malassezia, Rhodotorula, Geotrichum e Trichosporon do pelame e as cavidades naturais de cães e gatos sadios (Gambale et al., 1987; Cleff et al., 2007; Holanda et al., 2007). Leveduras do gênero Candida são agentes colonizadores e patógenos importantes, os quais podem ser encontradas na mucosa oral, trato gastrintestinal e vagina de indivíduos saudáveis. Essas leveduras não são habitualmente encontradas na microbiota residente da pele e, segundo a literatura, estão associadas a amplo espectro de doenças superficiais e invasivas, inclusive em cães aparentemente sadios (Colombo e Guimarães, 2003; Brown et al., 2005).

De acordo com trabalhos internacionais, Candida albicans pode, eventualmente, ser isolada da pele de cães e gatos sadios, porém em baixa frequência (9,4\%). Pesquisadores brasileiros obtiveram taxas iguais a $1 \%$ em cães portadores de dermatopatias diversas incluídos em um estudo realizado na cidade de Porto Alegre, RS. Outros observaram frequências um pouco mais elevadas (10\%) que aquelas, em cães que apresentavam lesões suspeitas de micose superficial na cidade de São Paulo. Em ambos os casos, os autores não puderam atribuir quaisquer lesões à $C$. albicans. Na presente investigação, esta foi a principal espécie isolada, mas sua patogenicidade tem sido associada a um contexto multifatorial, como caracteres raciais, idade e $\mathrm{pH}$ da pele do hospedeiro. Não foi possível estabelecer qualquer relação entre levedura isolada e a raça ou o sexo do animal na presente investigação; entretanto, esse aspecto deve ser futuramente investigado numa população mais significativa para que se possa evidenciar possível correlação (Gambale et al., 1987; Machado et al., 2004).

A colonização é considerada um importante fator de risco para a ocorrência de infecções oportunistas, uma vez que o agente infeccioso está em contato direto com o hospedeiro eventualmente susceptível. Segundo Colombo e Guimarães (2003), o agente etiológico de desordens invasivas em imunocomprometidos é, geralmente, o mesmo que coloniza o corpo do paciente. Apesar de os relatos existentes na literatura de infecção superficial e até sistêmica 
em cães trazerem dados consistentes a respeito, alguns clínicos desconsideram a pesquisa de leveduras no diagnóstico diferencial de algumas dermatopatias caninas (Costa et al., 1985; Machado et al., 2004). Um interessante estudo comparativo sobre a diversidade de isolados de C. albicans de origem humana e animal demonstrou a existência de genótipos hospedeiro-específicos e algumas linhagens espécie-específicas. No entanto, os autores ressaltam que os animais, principalmente os domésticos, podem desempenhar papel importante como reservatórios de $C$. albicans para seres humanos susceptíveis que mantêm contato próximo com esses animais (Edelmann et al., 2005).

Leveduras do gênero Rhodotorula, encontradas em fontes naturais e superfícies úmidas, também são integrantes da microbiota residente/transitória normal da pele de cães (Gambale et al., 1987; Cabañes et al., 1996; Lunardi et al., 2006). Segundo Holanda et al. (2007), Rhodotorula spp. têm sido apontadas como emergentes em quadros de infecção humana. A presença de Rhodotorula spp. é relatada com pouca frequência entre amostras clínicas provenientes de lesões de animais. Neste trabalho, ocorreu o crescimento de apenas um isolado de $R$. mucilaginosa, espécie apontada na literatura como agente etiológico de infecções invasivas em seres humanos (Lunardi et al., 2006).

Pode-se concluir que Candida albicans e Rhodotorula mucilaginosa são leveduras que compõem a microbiota do pelame de cães sadios que vivem em regime domiciliar (próximos aos proprietários) e que frequentam serviço de estética canina com regularidade. O conhecimento a respeito das espécies de leveduras que constituem a microbiota pode trazer futuros esclarecimentos a respeito do significado do isolamento de um agente microbiano comum, a partir de uma lesão, especialmente no caso de pacientes em condições clínicas especiais (Souza e Scarcelli, 2000; Machado et al., 2004). Partindo da premissa de que o estabelecimento da doença infecciosa é precedido pela colonização do hospedeiro susceptível pelo mesmo agente etiológico da infecção, é importante ressaltar que o estudo da microbiota constitui ferramenta bastante útil na monitoração de indivíduos imunossuprimidos, portanto susceptíveis às infecções oportunistas. Assim, o clínico veterinário deve considerar o emprego dessa ferramenta quando se deparar com casos especiais de animais imunologicamente anérgicos, levando sempre em conta que a obtenção apropriada da amostra clínica, o transporte adequado para o laboratório e o processamento imediato são condições essenciais para interpretação adequada do isolamento de um agente (Souza e Scarcelli, 2000). A alta frequência no isolamento de $C$. albicans obtida nessa investigação caracteriza exposição e risco de infecção tanto para os animais quanto para os proprietários susceptíveis que mantêm estreito contato com eles. O isolamento desses microrganismos de lesões em animais nessas condições deve ser avaliado criteriosamente.

Palavras-chave: cão, microbiota, leveduras, Candida albicans, Rhodotorula mucilaginosa

\begin{abstract}
Potentially pathogenic yeasts from the haircoat of dogs that live indoor and visit regularly an esthetic service were collected by the carpet technique applied on the fur. The microorganisms were isolated on Sabouraud dextrose agar supplied with chloramphenicol and identified by their morphological and biochemistry characteristics. Candida albicans was identificated in $95.2 \%$ of the canine population, followed by Rhodotorula mucilaginosa (4.8\%). It is known that $\mathrm{C}$. albicans is uncommon on health skin. By the other hand, the high frequency of this agent in this study may represent exposure and risk for opportunistic infections in dogs and other susceptible animals.
\end{abstract}

Keywords: dog, microbiota, yeasts, Candida albicans, Rhodotorula mucilaginosa

\title{
AGRADECIMENTOS
}

À Fundação de Amparo à Pesquisa do Estado de São Paulo (FAPESP), processo nº: 06/51677-7, e à Prof . Dra. Selene Dall'Acqua Coutinho, pela estima, pelo incentivo e pelo profissionalismo. 


\section{REFERÊNCIAS BIBLIOGRÁFICAS}

BENTUBO, H.D.L.; TOMAZ, M.A.; BONDAN, E.F. et al. Expectativa de vida e causas de morte em cães da região metropolitana de São Paulo (Brasil). Cienc. Rural, v.37, p.1021-1026, 2007.

BROWN, M.R.; THOMPSON, C.A.; MOHAMED, F.M. Systemic candidiasis in an apparently immunocompetent dog. J. Vet. Diagn. Invest., v.17, p.272-276, 2005.

CABAÑES, F.J.; ABARCA, M.L.; BRAGULAT, M.R. et al. Seasonal study of the fungal biota of the fur of dogs. Mycopathologia, v.133, p.1-7, 1996.

CLEFF, M.B.; XAVIER, M.O.; MARTINS, A.A. et al. Caracterizacion de la microbiota leveduriforme residente en la vagina de perras en diferentes fases del ciclo estral. Arch. Med. Vet., v.39, p.153-158, 2007.

COLOMBO, A.L.; GUIMARÃES, T. Epidemiologia das infecções hematogênicas por Candida spp. Rev. Soc. Bras. Med. Trop., v.36, p.599-607, 2003.

COSTA, E.O.; COUTINHO, S.D.; TEIXEIRA, C.M. et al. Dermatose por Candida albicans em cão. Rev. Microbiol., v.16, p.113-116, 1985.

DE HOOG, G.S.; GUARRO J.; GENÉ J. et al. Atlas of clinical fungi. 2.ed. Tarragona: Universitat Rovira i Virgili, 2000. 126p.
EDELMANN, A.; KRUGER, M.; SCHMID, J. Genetic relationship between human and animal isolates of Candida albicans. J. Clin. Microbiol., v.43, p.6164-6166, 2005.

GAMBALE, W.; CORRÊA, B.; PAULA, C.R. et al. Ocorrência de fungos em lesões superficiais de cães na cidade de São Paulo, Brasil. Rev. Fac. Med. Vet. Zootec., v.24, p.187-191, 1987.

HOLANDA, A.A.R.; FERNANDES, A.C.S.; BEZERRA, C.M. et al. Candidíase vulvovaginal: sintomatologia, fatores de risco e colonização anal concomitante. Rev. Bras. Ginecol. Obstet., v.29, p.3-9, 2007.

LUNARDI, L.W.; AQUINO, V.R.; ZIMERMAN, R.A. et al. Epidemiology and outcome of Rhodotorula fungemia in a tertiary care hospital. Clin. Infect. Dis., v.43, p.60-63, 2006.

MACHADO, M.L.S.; APPELT, C.E.; FERRERO, L. Dermatófitos e leveduras isoladas da pele de cães com dermatopatias diversas. Acta Sc. Vet., v.32, p.225-232, 2004.

MARIAT.F.; ADAM-CAMPOS, C. La technique du carré du tapis, méthode simple de prélevement dans les mycoses superficielles. $A n$. Inst. Pasteur, v.113, p.666-668, 1967.

SOUZA, C.A.I.; SCARCELLI, E. Agressão por microrganismos da microbiota endógena. Arq. Inst. Biol., v.67, p.275-281, 2000. 